\title{
Why neutrinos are different?
}

\author{
M. Libanov ${ }^{* \dagger}$ \\ Institute for Nuclear Research of the Russian Academy of Sciences, \\ 60th October Anniversary Prospect, 7a, 117312 Moscow, Russia \\ E-mail: mlems2.inr.ac.ru
}

\section{F.-S. Ling}

Institute of Theoretical Physics, Chinese University of Hong Kong

E-mail: fsling@phys.ufl.edu

\begin{abstract}
We present a short review of a 6-dimensional model where a flavour puzzle of the Standard Model fermions finds an elegant solution. The mechanism is based on an idea that the three fermionic generations originate from a single 6D family. The model explains in a natural way both charged fermions mass hierarchy and small mixings in the quark sector, and tiny neutrino masses and large neutrino mixings. We also discuss some phenomenological aspects of the model which can distinguish this class of the models from another one and can help to look for manifestations of new physics at colliders and other experiments.
\end{abstract}

The XXth International Workshop High Energy Physics and Quantum Field Theory September 24-October 1, 2011

Sochi Russia

${ }^{*}$ Speaker.

${ }^{\dagger}$ Many thanks to the Workshop organizers for the invitation. We wish to thank very warmly our colleagues J.M. Frere, E. Nugaev and S. Troitsky for ongoing collaboration. The work of M.L. has been supported in part by the Federal Agency for Science and Innovations under state contract 02.740.11.0244, by the grant of the President of the Russian Federation NS-5525.2010.2, by the RFBR grants 11-02-92108, and by the Dynasty Foundation. 


\section{Introduction}

One of the most intriguing issues of the Standard Model (SM) is a flavour puzzle which can be formulated as the following three problems:

- Problem of families replication and mass hierarchy: Why are there three families of fermions in the SM? In particular, why are these generations differing only by masses and why is this difference so large $\left(m_{\mathrm{top}} / m_{\mathrm{up}} \sim 10^{4}\right)$ ? Why are mixings in the quark sector relatively small and why is the mixing between first and third generations suppressed compare to the mixings between adjacent generations?

- Neutrino mass problem: Why do neutrinos have tiny masses and large mixings? Why neutrinos are so different from charged fermions?

- Flavour-changing neutral currents $(F C N C)$ problem: Why we do not observe "horizontal" inter-generation transitions?

This paper is a short review of existing works [1]-[9] In these papers in the frameworks of "large extra dimensions" (LED) (see Ref. [10] for a review) an elegant solution to the flavour puzzle has been suggested. The basic idea is an assumption that three generations of SM fermions appear as three zero modes of single multi-dimensional vector-like (with respect to SM gauge group) family. In the current review we concentrate on main points and basic ideas of the model at the cost of loss of mathematical rigor. For more complete and more advanced details the Reader is directed to the original works.

\section{The Setup}

Suppose one has single fermionic generation in a multi-dimensional theory. Let us consider a topological defect whose core corresponds to our four-dimensional world. Chiral fermionic zero modes may be trapped in the core due to specific interaction with the fields which build up the defect. In some cases, the index theorem guarantees that the number of chiral zero modes is determined by the topological number of the defect and by the charge of the fermion with respect to the symmetry group of the fields forming the defect. We use this property to obtain three fermionic generations localized on a defect while having only one generation in the bulk. If the Brout-EnglertHiggs scalar couples to the defect, it can also be trapped in the core. Hierarchy between masses of three fermionic modes arises due to their different profiles in extra dimensions $[1,2,3]$.

To be specific, let us assume that there are two large additional dimensions. ${ }^{1}$ The topological defect is an gauge vortex. A principal issue of models with LED is the localization of the SM gauge fields. One of possible ways to avoid this problem is to consider the transverse extra dimensional space as a compact manifold and to allow gauge fields to propagate freely in the extra dimensions. In what follows we assume that the extra dimensions form a two dimensional sphere with radius $R$ [4]. Though gravity is not included in the consideration, it should be stressed that the choice of

\footnotetext{
${ }^{1}$ The number of extra dimensions may be larger than two. What we in fact assume is that the size of another additional dimensions much smaller than the size of the two dimensions under consideration.
} 


\begin{tabular}{|c|c|c|c|c|c|c|}
\hline \multirow{2}{*}{\multicolumn{2}{|c|}{ Fields }} & \multirow[t]{2}{*}{ Profiles } & \multicolumn{2}{|c|}{ Charges } & \multicolumn{2}{|c|}{ Representations } \\
\hline & & & $U_{g}(1)$ & $U_{Y}(1)$ & $S U_{W}(2)$ & $S U_{C}(3)$ \\
\hline scalar & $\Phi$ & $\begin{array}{c}F(\theta) \mathrm{e}^{i \varphi} \\
F(0)=0, F(\pi) \simeq v\end{array}$ & +1 & 0 & 1 & 1 \\
\hline vector & $A_{\varphi}$ & $\begin{array}{c}A(\theta) / e \\
A(0)=0, A(\pi)=1\end{array}$ & 0 & 0 & $\mathbf{0}$ & $\mathbf{0}$ \\
\hline scalar & $X$ & $\begin{array}{c}X(\theta) \\
X(0)=v_{X}, X(\pi)=0\end{array}$ & +1 & 0 & 1 & 1 \\
\hline scalar & $H$ & $\begin{array}{c}H(\theta) \\
H_{i}(0)=\delta_{2 i} v_{H}, H_{i}(\pi)=0\end{array}$ & -1 & $+1 / 2$ & 2 & 1 \\
\hline fermion & $Q$ & $3 \mathrm{~L}$ zero modes & axial $(3,0)$ & $+1 / 6$ & 2 & 3 \\
\hline fermion & $U$ & $3 \mathrm{R}$ zero modes & axial $(0,3)$ & $+2 / 3$ & 1 & 3 \\
\hline fermion & $D$ & $3 \mathrm{R}$ zero modes & axial $(0,3)$ & $-1 / 3$ & 1 & 3 \\
\hline fermion & $L$ & $3 \mathrm{~L}$ zero modes & axial $(3,0)$ & $-1 / 2$ & 2 & 1 \\
\hline fermion & $E$ & $3 \mathrm{R}$ zero modes & axial $(0,3)$ & -1 & 1 & 1 \\
\hline fermion & $N$ & $\begin{array}{l}\text { Kaluza-Klein spectrum, } \\
\text { no zero mode }\end{array}$ & 0 & 0 & 1 & 1 \\
\hline $\begin{array}{r}\text { SM gauge } \\
\text { bosons }\end{array}$ & $\begin{array}{c}\gamma, G \\
Z, W^{ \pm}\end{array}$ & $\begin{array}{l}\text { Kaluza-Klein spectrum } \\
\text { starting from zero }\end{array}$ & 0 & - & - & - \\
\hline
\end{tabular}

Table 1: Field content of the model. For convenience, we describe here also the fields profiles in extra dimensions. $\theta$ and $\varphi$ are the polar and the azimuthal angles on the sphere, respectively. The vortex is localized at $\theta=0$.

the manifold is not important for our principal conclusions [5]. The extra dimensions can even be infinitely large. In this case, the role of the radius $R$ of the sphere is taken by a typical size of the localized gauge zero modes but not by a size of the extra dimensions.

The matter field content of the model is summarized in Table 1 and the profiles of the relevant fields are sketched in Fig 1. The scalar field $\Phi$, together with $U(1)_{g}$ gauge field, forms a vortex, while two other scalars, $X$ and $H$, develop profiles localized on the vortex. There is one fermionic generation which consists of five six-dimensional fermions $Q, U, D, L$, and $E$. Each of the fermions develops, in the vortex background, three chiral zero modes localized in the core of the vortex, which correspond to three generations of the SM fermions. There is an additional fermion $N$ which is neutral both under $U(1)_{g}$ and SM gauge group. This fermion is not localized and its Kaluza-Klein (KK) modes play a role of sterile neutrinos.

The spinor-scalar couplings, responsible for the localization of the fermionic zero modes, are $^{2}$

$$
\begin{gathered}
\sum_{\Psi=Q, L} g_{\Psi} \Phi^{3} \bar{\Psi} \frac{1-\Gamma_{7}}{2} \Psi+\text { h.c. } \longrightarrow 3 \text { left handed zero modes } \\
\sum_{\Psi=U, D, E} g_{\Psi} \Phi^{3} \bar{\Psi} \frac{1+\Gamma_{7}}{2} \Psi+\text { h.c. } \longrightarrow 3 \text { right handed zero modes }
\end{gathered}
$$

\footnotetext{
${ }^{2}$ We use the chiral representation for the six-dimensional Dirac $\Gamma$-matrices (see Ref. [1] for notations). In particular, $\Gamma_{7}=\operatorname{diag}(\mathbf{1},-\mathbf{1})$ is a six-dimensional analog of the four-dimensional $\gamma_{5}$.
} 


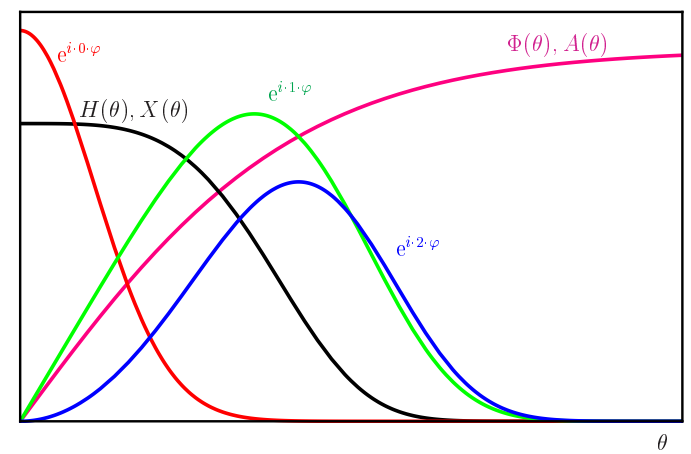

Figure 1: Sketch of the relevant background fields profiles (black and pink) and the fermionic zero modes (red, green and blue). The latter are labeled by their $\varphi$-dependencies.

This coupling may look surprising, as it is obviously non-renormalisable in 4 dimensions. We are not considering renormalisability of the theory here, as the 6-dimensional context is most likely an effective model, but even in this case it might be suitable that the 4-dimensional reduction be renormalisable.

What is important here is that three fermionic zero modes localized in the vortex background have different generalized (supplemented by $U(1)_{g}$ global rotations) angular momentum [4] and, as a result, have different $\varphi$ and $\theta$-dependencies. Typically, one has

$$
\Psi_{n}(\theta, \varphi) \sim f_{n}(\theta) \mathrm{e}^{i(3-n) \varphi}, \quad n=1,2,3,
$$

with the $\theta$-dependent wave functions $f_{n}(\theta)$ behaving near the origin as:

$$
f_{n}(\theta) \sim \theta^{3-n}, \quad \theta \rightarrow 0 .
$$

\section{Mass hierarchy and mixings}

The couplings (2.1) are responsible only for the localization of the fermionic zero modes. From the 4-dimensional point of view these modes describe localized massless chiral fermions with the usual quantum numbers under SM gauge group. Now comes the time to generate the "usual" fermion masses, and to break the $S U(3) \times S U(2) \times U(1)$ symmetry. This is done in the usual way, through the Brout-Englert-Higgs mechanism, at the price of introducing a scalar doublet, which we name $H$. In fact, for the purpose of separating the various quantum numbers, we write (e.g., for the "down" quarks), instead of the usual coupling:

$$
\mathscr{L}_{\text {Yukawa }}=Y_{d} H X \bar{Q} \frac{1-\Gamma_{7}}{2} D+\varepsilon_{d} Y_{d} H \Phi \bar{Q} \frac{1-\Gamma_{7}}{2} D+\text { h.c. },
$$

where $Y_{d}$ and $\varepsilon_{d} Y_{d}$ are coupling constants, the vortex scalar $\Phi$ has winding number 1 (see Table 1), while $X$ and $H$ have non-vanishing values at $\theta=0$ and zero winding number.

The reduction to 4 dimensions involves integration over $\varphi$ and $\theta$ and generates the mass terms. The first term in (3.1) yields

$$
m_{D, n m}^{(1)} \propto \int_{0}^{\pi} d \theta \sin \theta \int_{0}^{2 \pi} d \varphi f_{Q, n}^{\dagger} f_{D, m} X(\theta) H(\theta) \mathrm{e}^{i(n-m) \varphi} .
$$


Clearly, the integration over $\varphi$ guarantees that only diagonal entries occur. The integral over $\theta$ is saturated near the origin, more precisely, in the region where the fields $H$ and $X$ are non-zero. This region coincides, as it is shown in Fig. 1 , with the region $\left[0, \theta_{\Phi}\right]$ where $\Phi$ is appreciably different from its VEV [3, 6]. In this region one can safely use Eq. (2.3) for the fermionic wave functions and finds [4]

$$
m_{D, n m}^{(1)} \sim \delta_{n m} \sigma^{2(3-n)},
$$

where $\sigma=\theta_{\Phi} / \theta_{A}$ and $\theta_{A} \sim 1$ is the typical size of the gauge field of the vortex.

Due to the non-trivial $\varphi$-dependence of $\Phi$ the second term in (3.1) results in non-diagonal elements of the mass matrix:

$$
m_{D, n m}^{(2)} \sim \varepsilon_{d} \delta_{n+1, m} \sigma^{2(3-n)-1}
$$

The mass eigenstates are obtained by diagonalization (3.3), (3.4) and the power-like hierarchical mass pattern

$$
m_{33}: m_{22}: m_{11}=1: \sigma^{2}: \sigma^{4}
$$

arises at $\sigma \simeq 0.1$. The CKM-matrix has the form

$$
U^{\mathrm{CKM}} \sim\left(\begin{array}{ccc}
1 & \sigma & \sigma^{4} \\
\sigma & 1 & \sigma \\
\sigma^{2} & \sigma & 1
\end{array}\right)
$$

and reproduces observed mixings in the quark sector of the SM.

\section{Neutrinos masses. Why are they different?}

Now we want to consider whether the scheme can be extended to accommodate mass and mixing data in the neutrino sector. An obvious possibility to generate neutrino masses would be to treat them exactly like the charged fermions, with a Dirac mass obtained at the cost of introducing a $6 \mathrm{D}$ field $N$, bound to the vortex, and from which the three families of 4D right-handed neutrinos emerge. However, this possibility does not offer a natural explanation for the smallness of the neutrino masses, which in this case require tiny coefficients in the Lagrangian.

It is therefore tempting to consider other solutions, namely the case where the "right hande" neutrino field is NOT bound to the vortex. In the context of models with LED, tiny neutrino masses are often the result of a dilution effect: the field that provides right hande neutrinos, being singlet under the SM gauge group, can be non-localized, and therefore have a small overlap with the wave function of SM fields. A first attempt using a non-localized field $N$ was made in Ref. [7]. However, that approach predicts a neutrino mixing pattern that differs significantly from the observed one.

Here we consider another possibility [9]. We assume that spinor $N$ is a gauge singlet both under SM and $U(1)_{g}$ gauge group. It means first of all, that it can freely propagate in the extra dimensions. Secondly, one can (and, in general, have to) write a Majorana-like mass term in 6D for it:

$$
\frac{M}{2}\left(\bar{N}^{c} N+\bar{N} N^{c}\right)
$$


From 4-dimensional point of view one has KK tower of fermions possessing 4D Majorana mass $M$ and different Dirac masses starting from $1 / R^{3}$

Thirdly, the gauge invariance allows one to introduce the following couplings $\left(\tilde{H}=i \sigma_{2} H^{*}\right)$

$$
\sum_{S_{+}} Y_{v, S_{+}}^{+} \tilde{H} S_{+} \bar{L} \frac{1+\Gamma_{7}}{2} N+\sum_{S_{-}} Y_{v, S_{-}}^{-} \tilde{H} S_{-} \bar{L} \frac{1-\Gamma_{7}}{2} N+\text { h.c. }
$$

where $S_{+}$and $S_{-}$have $U(1)_{g}$ gauge charges 1 and -2 , respectively, and can be ${ }^{4}$

$$
\begin{aligned}
& S_{+}=X^{*}, \Phi^{*}, X^{* 2} \Phi, \ldots \\
& S_{-}=X^{2}, X \Phi, \Phi^{2}, \ldots
\end{aligned}
$$

In 4D these couplings give rise to mixings between heavy modes of $N$ and zero modes of active SM neutrinos. Together with the Majorana mass of modes of $N$ it winds up a "see-saw" mechanism yielding tiny Majorana masses of the active neutrinos. The resulting neutrino mass matrix can be schematically written in the form:

$$
m_{m n}^{v} \sim \int_{0}^{\pi} d \theta \int_{0}^{2 \pi} d \varphi F(\theta, \varphi) \bar{L}_{n}^{c} L_{m}
$$

where $F(\theta, \varphi)$ is determined by $S_{ \pm}$as well as by wave functions of $N$. The main point and the main difference from the quark sector (see Eq. (3.2)) is the presence charge conjugated spinor in the integrand: $\bar{L}^{c} \sim L^{T}$. This leads to completely different from (3.3), (3.4) selection rules. For instance, if we restricted ourselves by $\varphi$-independent $S_{ \pm}$(the first structures in (4.1)) then $F$ does not depend on $\varphi$, and one has

$$
m_{m n}^{v} \sim \int_{0}^{2 \pi} d \varphi \mathrm{e}^{i(4-n-m) \varphi} \sim \delta_{n, 4-m} \sim\left(\begin{array}{ccc}
\cdot & \cdot & 1 \\
\cdot & \sigma^{2} & . \\
1 & \cdot & \cdot
\end{array}\right)
$$

The inclusion of the $\varphi$-dependent structures in (4.1) gives rise to non-zero off secondary diagonal elements which have at least an order of $\sigma$.

What are consequences of the mass pattern (4.3)? The neutrino mass matrix (4.3) is diagonalized by a matrix with the structure

$$
U_{v} \sim\left(\begin{array}{ccc}
1 / \sqrt{2} & 1 / \sqrt{2} & \sigma \\
\sigma & \sigma & 1 \\
-1 / \sqrt{2} & 1 / \sqrt{2} & \sigma
\end{array}\right)+\mathscr{O}\left(\sigma^{2}\right)
$$

Let us emphasize that the large mixing angle in the 1-3 block is maximal up to $\sigma^{2}$ corrections. When the charged lepton mass matrix contains a large mixing angle in the 2-3 block, this model

\footnotetext{
${ }^{3}$ It is worth noting that a possible 6D Dirac mass term does not play any role and translates to a shift of 4D Dirac mass spectrum. This is the reason why we do not consider it.

${ }^{4}$ In the quark sector (Sec. 3) we restricted ourselves to considering $S_{+}=\Phi^{*}, X^{*}$ only. The reason is that an inclusion of more composite structures (e.g., $S_{-}$) does not play any significant role.
} 
predicts two large mixing angles, as observed. The remaining small mixing angle $U_{e 3}$, which corresponds to the weight of the lightest mass eigenstate in the electronic neutrino, is predicted to be of order $\sigma \sim 0.1$.

The diagonalized neutrino mass matrix has the inverted hierarchy pattern $\operatorname{diag}\left(m+\mathscr{O}\left(\sigma^{2}\right),-m+\right.$ $\left.\mathscr{O}\left(\sigma^{2}\right), m \sigma^{2}\right)$. Therefore, this model naturally predicts a hierarchy in the mass squared splittings relevant in neutrino oscillation experiments $\Delta m_{12}^{2} / \Delta m_{13}^{2} \sim \sigma^{2} \sim 0.01$, in good agreement with the observed data $\Delta m_{12}^{2} / \Delta m_{13}^{2} \simeq 3.2 \%$. Moreover, $m_{1}$ and $m_{2}$ form a "pseudo-Dirac" pair as $m_{1}+m_{2} \sim \sigma^{2} m$. It leads to a partial cancellation in the effective Majorana mass, $\left|\left\langle m_{\beta \beta}\right\rangle\right|=$ $\left|\sum_{i} m_{i} U_{e i}^{2}\right| \simeq 1 / 3 \sqrt{\Delta m_{13}^{2}}$, defining the amplitude for neutrinoless double-beta decay.

\section{FCNC}

From the 4D point of view the presented model completely reproduces all properties of SM if one considers zero modes only (including zero modes of the SM gauge fields). In particular, all FCNC processes are strongly suppressed as it occurs in SM. However, from the 6D point of view we have only a single generation and there is no difference, say, between $\mu$ and $e$. That is, heavy (non-zero) modes of the neutral SM gauge bosons can (and have to) violate flavour and/or lepton numbers. Nevertheless, without account of inter-generation mixings, the generalized angular momentum or, what is the same, the generation number $G$ is exactly conserved. This forbids all processes with nonzero change of $G$; the probabilities of the latters in the full theory are thus suppressed by powers of the mass-matrix mixing parameter, $(\varepsilon \sigma)^{2 \Delta G}$. However, the amplitudes of processes with $\Delta G=0$ but lepton and quark flavours violated separately are suppressed only by the mass squared of the KK modes of the SM gauge bosons.

In Ref. [5] the following specific flavour violating processes have been studied:

- $\Delta G=0: K_{L}^{0 \rightarrow} \rightarrow \mu e, K^{+} \rightarrow \pi^{+} e^{-} \mu^{+}$;

- $\Delta G=1: \mu \rightarrow e \gamma, \mu \rightarrow 3 e, \mu \rightarrow e$-conversion;

- $\Delta G=2: K_{L}-K_{S}$ mass difference and CP violation in kaons.

These processes are known to give the strongest constraints on masses and couplings of new vector bosons. It was found that indeed the pattern of flavour violation is distinctive: contrary to other models, processes with change of the generation number $G$ by one or two units are strongly suppressed compared to other rare processes. The strongest constraint on the model arises from non-observation of the decay $K^{0} \rightarrow \mu e$; it requires that the size of the sphere (size of the gaugeboson localization) $R$ satisfies $\varkappa / R \gtrsim 64 \mathrm{TeV}$, where $\varkappa$ is a dimensionless parameter depending on specific model: details of the geometry, mechanism of the localization of the vector fields, and so on. A clear signature of the model would be an observation of $K^{0} \rightarrow \mu e$ decay without observation of $\mu \rightarrow 3 e, \mu \rightarrow e \gamma$ and $\mu e$-conversion at the same precision level.

For the spherical model under discussion $\varkappa=1$. However, in general one can expect that $\varkappa$ can differ from unit and can be small enough $(\varkappa \sim 0.01)$. In the latter case the masses of the first non-zero excitations of the SM gauge bosons $M^{\prime} \simeq 1 / R$ can be of order of few $\mathrm{TeV}$ and vector bosons can, in principle, be observed at colliders. In general, there are two possibilities. First of 
all, one can try to search for "usual" heavy vector bosons, that is the heavy KK modes which do not change generation number. The second possibility is to look for heavy KK vector bosons due to the flavour-changing decay modes into $(\mu e)$ or $(\mu \tau)$ pairs. The flavour-changing decays of this kind have a distinctive signature: antimuon and electron (or their antiparticles) with equal and large transverse momenta in the final state.

The latter possibility has been investigated in Ref. [8]. In particular, it was found for the expected LHC value of $100 \mathrm{fb}^{-1}$ for luminosity and $\sqrt{s}=14 \mathrm{TeV}$ that the number of $p p \rightarrow \mu^{+} e^{-}$ events varies from 1 to 10 per year for $M^{\prime} \simeq 3 \div 1 \mathrm{TeV}$. The probability of the production $\left(\mu^{-} e^{+}\right)$ pairs is approximately ten times smaller due to the former process can use valence $u$ and $d$-quarks in the proton, while the second only involves partons from the sea. The same numbers are representative also for the $(\mu \tau)$ channels.

There are also other signatures of FCNC effects, in particular, with hadronic final states, when $(\bar{t}, c)$ or $(\bar{b}, s)$ jets are produced. The dominant contribution to these processes arises from the interactions with higher KK modes of gluons, which have large coupling constant. For the mass of $M^{\prime}=1 \mathrm{TeV}$ the number of events has been estimated as $N=1.2 \cdot 10^{3}$. But potentially large SM backgrounds should be carefully considered for such channels.

\section{Conclusions}

To conclude, we presented a possible elegant solution to the flavour puzzle. The mechanism is based on an assumptions that the three SM generations originate from a single family in a higherdimensional theory. The generation number is none other than angular momentum and, therefore, has an geometrical origin.

We explained why neutrinos are different from the sector of the charged fermions. A light neutrino mass matrix where one mixing angle is automatically maximal and where the eigenvalues obey an inverted hierarchy with a pseudo-Dirac pattern for the heavier states $m_{1} \simeq-m_{2} \gg m_{3}$ is a result of a mixing between active neutrinos and a single heavy sterile $6 \mathrm{D}$ fermion with Majoranalike mass.

The 6D Lagrangian with one generation contains much less parameters than the effective one. All masses and mixings of the SM fermions are governed by a few parameters of order one. This fact allows for specific phenomenological predictions. In particular, the KK modes of the vector bosons mediate flavour-violating processes. The pattern of flavour violation is distinctive: contrary to other models, processes with change of the generation number by one or two units are strongly suppressed compared to other rare processes. The strongest constraint on the model arises from non-observation of the decay $K \rightarrow \mu e$; it requires that the size of the extra-dimensional sphere (size of the gauge-boson localization) satisfies $1 / R \gtrsim 64 \mathrm{TeV}$. The $\mathrm{KK}$ modes of vector bosons have larger masses, but for large enough $R$, could be detected by precision measurements at colliders.

One more point which we did not discuss in this review is a Brout-Englert-Higgs boson properties. The model predicts that its mass can not be much larger then $100 \mathrm{GeV}$ [6].

\section{References}

[1] M. V. Libanov and S. V. Troitsky, "Three fermionic generations on a topological defect in extra dimensions,” Nucl. Phys. B 599, 319 (2001) [hep-ph/0011095]. 
[2] J. M. Frere, M. V. Libanov and S. V. Troitsky, "Three generations on a local vortex in extra dimensions,” Phys. Lett. B 512, 169 (2001) [hep-ph/0012306].

[3] M. V. Libanov and E. Y. Nougaev, "Towards the realistic fermion masses with a single family in extra dimensions,” JHEP 0204, 055 (2002); Surveys High Energ. Phys. 17 (2002) 165 [hep-ph/0201162].

[4] J. M. Frere, M. V. Libanov, E. Y. Nugaev and S. V. Troitsky, "Fermions in the vortex background on a sphere,” JHEP 0306, 009 (2003) [hep-ph/0304117].

[5] J. M. Frere, M. V. Libanov, E. Y. Nugaev and S. V. Troitsky, "Flavor violation with a single generation,” JHEP 0403, 001 (2004) [hep-ph/0309014].

[6] M. V. Libanov and E. Y. Nugaev, "Properties of the Higgs particle in a model involving a single unified fermion generation,” Phys. Atom. Nucl. 70, 864 (2007) [Yad. Fiz. 70, 898 (2007)] [arXiv:hep-ph/0512223].

[7] J. M. Frere, M. V. Libanov and S. V. Troitsky, "Neutrino masses with a single generation in the bulk," JHEP 0111, 025 (2001) [hep-ph/0110045].

[8] J. M. Frere, M. V. Libanov, E. Y. Nugaev and S. V. Troitsky, "Searching for family number conserving neutral gauge bosons from extra dimensions,” JETP Lett. 79, 598 (2004) [Pisma Zh. Eksp. Teor. Fiz. 79, 734 (2004)] [arXiv:hep-ph/0404139].

[9] J. M. Frere, M. Libanov and F. S. Ling, "See-saw neutrino masses and large mixing angles in the vortex background on a sphere,” JHEP 1009, 081 (2010) [arXiv:1006.5196 [hep-ph]].

[10] V. A. Rubakov, “Large and infinite extra dimensions: An Introduction,” Phys. Usp. 44, 871 (2001) [Usp. Fiz. Nauk 171, 913 (2001)] [arXiv:hep-ph/0104152]. 